\title{
Medicina personalizada, individualizada, de precisión y centrada en el paciente; diferencias o sinónimos. Su importancia
}

\author{
Personalized, individualized, precision, and patient-centered \\ medicine; differences or synonyms. Its importance
}

\author{
Federico Leopoldo Rodríguez Weber, José Luis Ramírez Arias
}

Citar como: Rodríguez WFL, Ramírez AJL. Medicina personalizada, individualizada, de precisión y centrada en el paciente; diferencias o sinónimos. Su importancia. Acta Med GA. 2022; 20 (1): 111-112. https://dx.doi.org/10.35366/103572

Son muchas las ideas que se pueden exponer en relación a términos como medicina individualizada, personalizada, medicina de precisión o centrada en el paciente, por ejemplo, algunos de los conceptos tienen su origen en la creencia de que los individuos son poseedores de características únicas en los niveles moleculares, genéticos, fisiológicos, culturales, de exposición ambiental y del comportamiento, por tanto no suena ilógico que se necesite de diferentes conceptos para la atención de las enfermedades que presenten adaptándola a las características matizadas de cada individuo. Esta forma de pensar se fundamenta hasta cierto punto como consecuencia de los avances tecnológicos, científicos y de investigación que nos permiten contar con la secuenciación del genoma y de los conceptos vigentes del ácido desoxirribonucleico (ADN), de la proteómica y de los protocolos de imagen y dispositivos de monitoreo relacionados con la salud, los cuales han revelado una gran variación entre cada individuo en los diferentes procesos de enfermedad.

\footnotetext{
* Médico Internista. Profesor adjunto de la Residencia de Medicina Interna. Facultad Mexicana de Medicina de la Universidad La Salle. Coordinador de Pregrado y Postgrado, Hospital Angeles Health System.

₹ Médico Radiólogo. Director Médico. Miembro de la Academia Nacional de Medicina y la Academia Mexicana de Cirugía.

Hospital Angeles Pedregal. Ciudad de México.
}

Correspondencia:

Dr. Federico Leopoldo Rodríguez Weber

Correo electrónico: fweber@saludangeles.com

Aceptado: 10-07-2021.

www.medigraphic.com/actamedica
La medicina centrada en el paciente postula que hay que enfatizar la atención individual de cada uno de los enfermos, con un enfoque de filosofía holística, no fragmentada del ser humano en todas sus dimensiones anatomo-clínicas y biopsicosociales, no dejando que la influencia tecnológica y la súper especialización dividan o afecten el ver al paciente en forma integral. Se debe tener claro el reconocimiento del principio y el derecho al individuo de hacerlo partícipe de las decisiones relacionadas con su salud, permitiendo que él sea el centro de las acciones médicas. "La medicina centrada en el paciente debe tener en cuenta los objetivos, los valores, las creencias y las preferencias de los enfermos, pero para ello se requiere una amplia y eficiente comunicación médico-paciente para asegurar que el enfermo tenga una participación real en las decisiones médicas que lo afectan directamente".

La medicina de precisión se ha promovido como un enfoque para individualizar el diagnóstico y el tratamiento de enfermedades a partir de características genéticas, de la presencia o ausencia de biomarcadores, de la presencia de fenotipos específicos y características biopsicosociales. ${ }^{2}$ Actualmente el coto de la medicina de precisión es muy alto, seguramente por la tecnología con la que está vinculada, y no sólo eso, pues no se cuenta con evidencia de calidad para medir su efectividad en muchas aplicaciones. Para que el potencial de la medicina de precisión trascienda, deberá contar con los principios de la medicina basada en evidencia, recordando que la evidencia aislada no es suficiente para tomar decisiones clínicas, que éstas deben ser aplicadas de acuerdo con los valores y preferencias del paciente teniendo en consideración los recursos con los que se cuenta, así como la jerarquía de evidencias que permiten orientar la atención clínica. ${ }^{3}$ 
De nada sirve contar con toda la tecnología y todo el conocimiento si no tenemos además un comportamiento del médico o del grupo de trabajo acompañado de valores en los que resalten la veracidad, la honestidad, el compromiso de una atención médica realizada con alto sentido de profesionalismo, lo que permite asegurar una atención de calidad y segura con los recursos existentes, lo que da certeza al paciente de estar recibiendo la mejor atención posible. Lo anterior apoya, además de ser un ejemplo, al resto de los profesionales al vincular la educación continua con estos avances científicos. ${ }^{4}$

Dar atención individualizada a cada paciente reconociendo sus antecedentes, riesgos ambientales, antecedentes patológicos, estrato sociocultural y económico así como su estado de salud, junto con subrayar la parte ética y humanística, harán exitosa la atención integral de los pacientes, pero es esencial fortalecer la educación de los futuros médicos enfatizando los ejes éticos y humanísticos, lo que se debe considerar como una verdadera necesidad ante la observación de conductas inapropiadas de algunos profesionales de la salud. Esta enseñanza debe ir acompañada de una adecuada teoría del desarrollo moral del hombre y en particular, de asegurar una reflexión crítica en el sustento de tal formación, para ello es necesario el compromiso de desarrollo y evaluación de los profesores en estos temas que son de la práctica médica diaria, en los procedimientos y en los escenarios utilizados para este fin.

Por último, se debe recalcar la importancia de ver al paciente como un ser completo integral, poseedor de una estructura anatómica con órganos y sistemas, sujetos a sufrir enfermedades o disfunciones, pero que por el instinto, la voluntad, los sentimientos, la percepción y el deseo de curarse con médicos que, a través de su ejercicio profesional, sean capaces de sumar e integrar la información clínica su- ficiente para ofrecer una atención adecuada integral. Puede sonar contradictorio, por tanto, la necesidad de contar con especialistas, pues es la especialidad la que con frecuencia fracciona la atención integral del individuo. La anterior situación se genera por la gran cantidad de información que se puede recabar con el conocimiento en un área, por eso la propuesta es que siempre sea un médico integrador o se cuente con médicos integradores que desarrollen una estrecha relación entre el médico integrador y el médico especialista, centrando el conjunto de conocimientos y conclusiones en la realidad y en las características de ese paciente en específico. ${ }^{5}$

Por tanto, mientras no existan definiciones universalmente aceptadas del significado real de la medicina personalizada, de la medicina individualizada, de la medicina enfocada en el paciente, y de la medicina de precisión, continuaremos utilizado los términos según la definición de cada autor, pero sin lugar a duda lo que sí debe quedar claro para cada uno de los profesionales de la salud, es la importancia de considerar al paciente como un individuo integral, de tal forma que todas las esferas que lo conforman sean tomadas en cuenta en una atención médica altamente profesional.

\section{REFERENCIAS}

1. Fahey T, NicLiam B. Assembling the evidence for patient centred care. BMJ. 2014; 349: g4855.

2. Sánchez E. ¿Qué es la medicina de precisión? [Internet]. MejorconSalud. 2021. Disponible en: https://mejorconsalud.as.com/ medicina-precision/

3. Chow N, Gallo L, Busse JW. Evidence-based medicine and precision medicine: complementary approaches to clinical decision-making. Prec Clin Med. 2018; 1 (2): 60-64.

4. Rodríguez WFL. Valores, la medicina y la docencia. Acta Med. 2021; 19 (1): 7-8.

5. Suárez Cuba MA. Medicina centrada en el paciente. Rev Méd La Paz. 2012; 18 (1): 67-72. 